\title{
Minimum Energy Accumulative Routing in Wireless Networks
}

\author{
Jiangzhuo Chen, Lujun Jia, Xin Liu, Guevara Noubir, Ravi Sundaram \\ College of Computer and Information Science \\ Northeastern University \\ Boston, MA, 02115 \\ \{chenj, lujunjia, liux, noubir, koods\}@ccs.neu.edu
}

\begin{abstract}
In this paper, we propose to address the energy efficient routing problem in multi-hop wireless networks with accumulative relay. In the accumulative relay model, partially overheard signals of previous transmissions for the same packet are used to decode it using a maximal ratio combiner technique [1]. Therefore, additional energy saving can be achieved over traditional energy efficient routing. The idea of accumulative relay originates from the study of relay channel in information theory with a main focus on network capacity. It has been independently applied to minimum-energy broadcasting in [2], [3].

We formulate the minimum energy accumulative routing problem (MEAR) and study it. We obtain hardness of approximation results counterbalanced with good heuristic solutions which we validate using simulations. Without energy accumulation, the classic shortest path (SP) algorithm finds the minimum energy path for a source-destination pair. However, we show that with energy accumulation, the SP can be arbitrarily bad. We turn our attention to heuristics and show that any optimal solution of MEAR can be converted to a canonical form - wavepath. Armed with this insight, we develop a polynomial time heuristic to efficiently search over the space of all wavepaths. Simulation results show that our heuristic can provide more than $30 \%$ energy saving over minimum energy routing without accumulative relay. We also discuss the implementation issues of such a scheme.
\end{abstract}

Keywords: ad hoc and sensor networks, optimization, simulation, graph theory.

\section{INTRODUCTION}

A wireless ad hoc network or sensor network consists of a collection of geographically dispersed nodes that usually communicate using radio frequency links. In many cases the nodes are operated by batteries with limited, non-replenishable energy. These nodes are supposed to be operational for a long period of time in an unattended manner. This means that the network's operational lifetime is determined by the lifetime of the battery. Therefore, energy efficiency is a critical factor in the design of such networks in order to prolong the lifetime of the network.

In this paper, we consider using an interesting property of wireless networks, which is partial overhearing, to save transmission energy in multi-hop communications. One can assume that, within a certain range, the neighboring nodes can receive and correctly decode the received packet. Neighboring nodes within a larger range can only detect and acquire the timing synchronization of the packet while not being able to correctly decode the whole packet. The threshold for detection is usually set to be a few decibels higher than the noise floor in commercial devices. Thus it allows nodes to partially overhear packets within a range of 5 to 10 times the normal transmission range. Note that several commercial chips already offer multiple data rates depending on the received energy. For example RF Monolithics ${ }^{1}$ transceivers can receive (with BER $<10^{-3}$ ) at $-106 \mathrm{dBm}$ for $2.4 \mathrm{Kbps}$ and $-97 \mathrm{dBm}$ for $115 \mathrm{Kbps}$. Most IEEE802.11 cards operate within a large sensitivity range depending on data rates (e.g., Cisco 350 cards operate within $[-94 \mathrm{dBm},-71 \mathrm{dBm}]$ for rates within [1, 54Mbps]). Therefore, if the packet header uses a strong modulation/coding scheme it allows far away nodes to collect packets with some of the bits in the packet payload in error. Using a maximal ratio combiner [1], multiple partially overheard copies of the same packet would enable the receiver to fully decode the packet. This scheme forms the basis of energy saving in our new model. We refer to this mode of communication as the Accumulative Relay $(A R)$ model.

An efficient use of the $A R$ requires limited interference from concurrent sessions. We assume that the network operates in the wideband power limited regime with no co-channel interference. This regime is realistic for some wireless networks, especially sensor networks and ultra-wideband communication. Sensor networks have extreme limitations in energy and sufficient large frequency bandwidth. Furthermore, in many sensor network applications, the traffic load is low and the nodes are in the sleep or idle mode most of the time. This is because the nodes only need to respond to infrequent events or

\footnotetext{
${ }^{1}$ TR100 Hybird Transceiver http://www.rfm.com.
} 
queries. Such low load also justify the no co-channel interference assumption.

Related Work: Communication is typically the most expensive activity of a wireless node in terms of energy consumption [4], [5]. Various techniques have been proposed to reduce the energy expenditure in the communication procedure. Among them energy efficient routing is an important branch which received significant attention during the past years. Energy efficient routing considers the problem of identifying energy efficient paths in wireless networks based on various metrics [6]-[10]. Rodolplu et al. [8] minimize the end to end aggregate energy consumption. Chang et al. [7] aim at maximizing the network operational lifetime. Banerjee et al. [9] add link error rate to the metric besides transmission energy consumption and try to minimize the energy cost for a reliable communication. More research results in the field can be found in [11]-[15]. All these schemes are studied under what we call the traditional multi-hop model (TM).

In the $T M$ model, sending one unit of information from node $A$ to node $B$ requires a transmission power at least equal to the receiving threshold divided by the channel gain from $A$ to $B$. This places a lower bound on the total energy consumption, under the $T M$ model, given the amount of information needed to be transmitted and the network topology. The essential difference between the $A R$ and $T M$ model is that nodes in $A R$ model do not discard unsuccessfully received broadcasting packets as they do in the $T M$ model. The partially overheard packet, referred to as leakage in the paper, contributes to the final reception of the packet at the intended receiver in the $A R$ model. This leads to further energy saving over the optimal energy schemes in the TM model.

Our work originates from the relay channel which was introduced and studied from an information theoretic perspective by the information theory community [16], [17]. The focus of the relay channel is to transmit information from the source to the destination as efficiently as possible with the cooperation from the relays [17]. Previous research on the relay channel mainly focused on theoretical capacity issues [16], [18]-[22]. We are interested in developing constructive strategies and efficient algorithms for a practical use of the relay channel concept. Maric et al. [3] and Agarwal et al. [2] propose to use the idea of energy accumulation to reduce the energy cost of broadcast in wireless networks. We investigate the multi-hop unicast scenarios in this paper.

Setup and Contributions: In this paper, we introduce the problem of minimum energy unicast routing in a wireless network using the $A R$ model. We prove that if the energy spent by each node in the relaying process is upper bounded by some fixed value, then identifying the minimum energy routing schedule is an NP-complete problem. We introduce the notion of wavepath (a canonical form of accumulative relaying) and show that any minimum energy relay routing schedule can be transformed into a wavepath that has the same energy cost. Therefore we can focus on finding a minimum energy wavepath. The hardness of determining the minimum energy wavepath lies in identifying the participating nodes in the schedule, and not in finding the order, as it is the case in the problem of broadcast with energy accumulation [3]. We develop a heuristic to find an energy efficient wavepath. We simulate our heuristic and show that it provides significant energy saving compared to the traditional shortest path algorithms which gives the optimal energy paths in TM networks (i.e., above 30\%). In order to better characterize the theoretical difficulty and value to the accumulative approach, we show that for a general channel propagation model, the cost of the optimal wavepath can be asymptotically smaller than that of the energy efficient path found by the shortest path algorithms. Therefore an optimal relaying strategy can provide arbitrarily better performance than classical shortest path.

The rest of the paper is organized as follows: we introduce the network model in Section II; we formulate the Minimum Energy Accumulative Routing problem and propose a heuristic with bounded performance guarantee in Section III; the simulation results which show significant energy saving over traditional routing techniques is provided in Section IV; at last we discuss implementation issues in Section V.

\section{NeTWORK MODEL}

We consider a wireless network with $N$ nodes using omni-directional antennas. Each node can dynamically tune its transmission power from zero to the maximum power level $p_{\max }$. The network is static and the traffic within the network is unicast. The bandwidth is sufficiently large compared to the traffic load. We study the aggregate transmission energy consumption to successfully transmit a packet from the source to its destination under the $A R$ model.

The wireless link between two nodes $i$ and $j$ is modeled using the channel gain $g_{i, j}$. In the general graph model the channel gain can take arbitrary values. We also consider the commonly used geometric propagation model with $g_{i, j}=\frac{1}{C d_{i, j}^{\alpha}}$ [23] where $d_{i, j}$ denotes the distance between node $v_{j}$ and node $v_{i}, \alpha$ denotes the power attenuation (path loss) exponent taking values between 2 and 4 depending on the environment, and $C$ is a constant and depends on the antenna gains and operation frequency. Without loss of generality, $C$ is normalized to be 1 . Let $p_{i}$ denote the transmission power 
at $v_{i}$, and $p_{j}^{r}$ denotes the received signal power at $v_{j}$. We have $p_{j}^{r}=p_{i} g_{i, j}$. Under both $T M$ and $A R$ models, for a packet to be correctly decoded the $p_{j}^{r}$ must exceed a threshold $H$ (assuming constant noise level and no cochannel interference). A packet received at a power level less than the threshold cannot be correctly decoded. We refer to such packets as leakage from a transmission.

\section{A. Power Consumption in the AR Model and the TM Model}

Let a sequence of nodes $\left[v_{1}, v_{2}, \cdots, v_{w}\right]$ be the path from the source $v_{1}$ to the destination $v_{w}$. Under $T M$, each node on the path needs to transmit at a power level $p_{i}$ at least $H / g_{i, j}$ for the transmission to be correctly received by the next hop and forwarded toward the destination. The total transmission energy consumption of the path under the $T M$ model is at least:

$$
\sum_{i} p_{i}=\sum_{i} H / g_{i, i+1}
$$

Now let us examine the transmission energy consumption of the path under the $A R$ model. Note a node is allowed to transmit the packet only after it correctly decodes the packet, except for the source.

For a multi-hop unicast communication under the $A R$ model, the same packet is transmitted by each node on the path sequentially. All the nodes except the source and the first hop can get multiple leakages from previous transmissions, and thus accumulate energy from them. Let $l_{i+1}$ denote the total energy accumulated from the leakages on node $v_{i+1}$; we have $l_{i+1}=$ $\sum_{v_{j} \in\left\{v_{1}, \cdots, v_{i-1}\right\}} p_{j} g_{j, i+1}$. Hence, for the transmission from $v_{i}$ to $v_{i+1}$ to be correctly decoded, the received signal power plus the leakages already accumulated at $v_{i+1}$ needs to exceed $\mathrm{H}$. In other words, $v_{i}$ needs to send the packet at power level of $\left(H-l_{i+1}\right) / g_{i, i+1}$. Thus the total energy consumption for the path under $A R$ model is:

$$
\sum_{i} p_{i}=\sum_{i}\left(H-l_{i+1}\right) / g_{i, i+1}
$$

It is easy to see that each item in the Equation (2) is less than that of Equation (1), since the leakage energy is non-negative. Therefore, the total energy consumption of a given path in $A R$ is less than that of $T M$.

Due to the limited computational power and memory space of wireless nodes, the number of leakages a node can accumulate is usually restricted. Also in a real wireless network, nodes cannot detect a signal with arbitrary small power level. Thus, in our subsequent sections, we will also consider a restricted model of accumulative routing, $k$-Relay, where a wireless node can only accumulate energy from the last $k$ transmissions of the same packet. It is easy to see that $A R$ routing is actually a generalization of $T M$ routing. Since in the $T M$ model, each node decodes the packet only based on the latest transmission, which is exactly 1-Relay in the $A R$ model.

\section{B. Motivations for AR Routing}

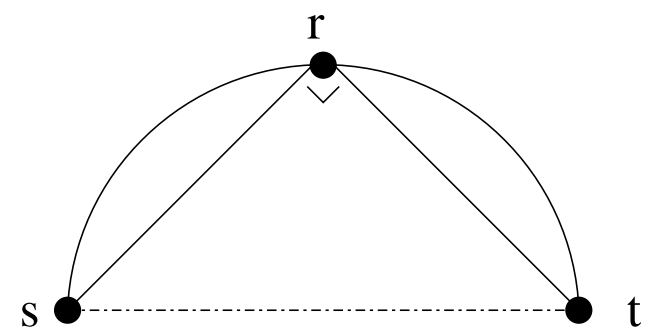

Fig. 1. For triangle $\triangle s r t, d_{s, r}=d_{r, t}=1$ and $\angle s r t=90^{\circ}$. Clearly, $s \rightarrow r \rightarrow t$ in the $T M$ model consumes the same amount of energy as $s \rightarrow t$. While with $A R$ routing, $25 \%$ energy saving can be achieved, since $s$ 's transmission to $r$ has a leakage at $t$. $S$

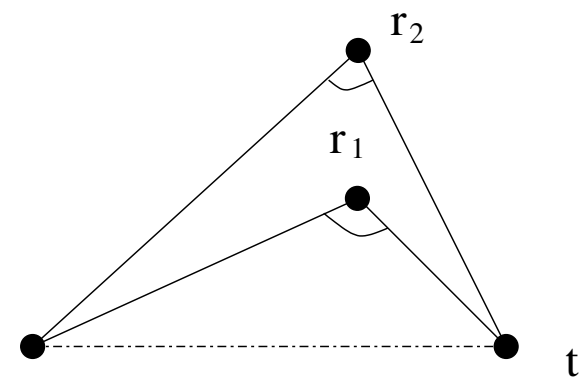

Fig. 2. Node $r_{1}$ has $\angle s r_{1} t>90^{\circ}$ and node $r_{2}$ has $60^{\circ}<$ $\angle s r_{2} t<90^{\circ}$. To achieve energy saving, only $r_{1}$ can act as an intermediate node between source $s$ and destination $t$ in $T M$ routing; while in $A R$ routing, $r_{2}$ can also act as an intermediate node.

We now discuss the minimum energy routing problem under two different models: $T M$ and $A R$. Assume the power attenuation exponent $\alpha$ equals to 2 in the following examples. The first motivation for using $A R$ routing is that it provides a new means of energy saving. In the $T M$ model, the traditional shortest path algorithm can find the minimum energy path for the given source and destination, which places the lower bound for the transmission energy consumption. With $A R$ routing, however, the energy consumption can be less than this lower bound. In Figure 1, under the $T M$ model, the path $s \rightarrow r \rightarrow t$ consumes the same amount of energy, $2 H$, as $s \rightarrow t$, since $\angle s r t=90^{\circ}$. With $A R$ routing, $s$ 's transmission at power level $H$ yields a successful packet decoding at $r$, since $g_{s, r}=1 / d_{s, r}=1$. At the same time, this transmission also yields a leakage 
of $H / 2$ on $t$, since $d_{s, t}=1 /(\sqrt{2})^{2}=1 / 2$. Thus, node $r$ only needs to transmit (to $t$ ) at power level $(H-H / 2) / d_{r, t}^{2}=H / 2$. The total energy consumption for transmitting a packet from $s$ to $t$ is only $3 / 2 H$ in $A R$ routing. This leads to a $25 \%$ energy saving over routing under TM model.

Another advantage of routing under the $A R$ model is that more nodes could act as intermediate nodes between the source and the destination to help forwarding the packet. Figure 2 shows the difference of the possible relay region between $A R$ and $T M$ routing.

\section{Minimum Energy Accumulative Routing}

In this section, we give the mathematical formulation of the minimum energy accumulative routing problem. We study the problem in the general graph model first, where link gains can take arbitrary values. We prove that with a cap on the transmission power the problem is NP-complete and show that the shortest path heuristic can be arbitrarily bad compared with the optimal solution. We also prove that the optimal solution satisfies the wavepath property. We propose a polynomial time heuristic RPAR. Last, we derive a lower bound of energy consumption in the $k$-Relay scenario.

\section{A. Problem Formulation}

Given source $s$ and destination $t$, a transmission schedule $S=\left[\left(v_{1}, p_{1}\right), \cdots,\left(v_{w}, p_{w}\right)\right]$, where $v_{i} \in V$ and $p_{i} \geq 0$ is the transmission power of nodes $v_{i}$, is feasible for $(s, t)$ if:

1) The source is the first transmitter and the destination is the ultimate receiver, i.e., $v_{1}=s, v_{w}=t$.

2) Every node in the schedule except $v_{1}$ has to first correctly decode the packet before being able to transmit it. For the general $A R$ routing where there is no restriction on the relay level,

$$
\forall i>1, \quad \sum_{j=1}^{i-1} p_{j} g_{j, i} \geq H
$$

for the $k$-Relay case,

$$
\forall i>1, \quad \sum_{j=i-k}^{i-1} p_{j} g_{j, i} \geq H,
$$

where $g_{j, i}$ is the channel gain from $v_{j}$ to $v_{i}$.

So a feasible transmission schedule is an ordered list of node ID, transmission power pairs. Starting from the source, each node needs to transmit with enough power such that the next node collects, from previous transmissions, a total amount of energy at least the receiving threshold.
Definition 1: The Minimum EnERgy ACCUMulaTIVE RoUTING problem $\operatorname{MEAR}(V, s, t)$ looks for a feasible transmission schedule $S=\left[\left(v_{1}, p_{1}\right), \cdots,\left(v_{w}, p_{w}\right)\right]$ for $(s, t)$, such that the total transmission energy $E(S) \triangleq$ $\sum_{i=1}^{w} p_{i}$ is minimized.

\section{B. Complexity of The Problem}

In the following, we show that the general graph version of the MEAR problem is NP-complete when there is a cap on the amount of energy one node can spend for one packet. We prove the NP-completeness of MEAR by a reduction from the SET COVER (SC) problem. It is well known that the SC problem is NPcomplete, and is not approximable within $(1-\varepsilon) \ln V$ for any $\varepsilon>0$, where $V$ is the size of the set, unless $\mathrm{NP} \subset \mathrm{DTIME}\left(V^{\log \log V}\right)$ [24]. Thus, for a general graph with arbitrary link gains and a limited energy budget, there does not exist an approximation algorithm for MEAR with an approximation ratio less than $O(\ln N)$, where $N$ is the number of nodes.

Theorem 1: The $\operatorname{MEAR}(V, s, t)$ problem is NPcomplete for a general graph with arbitrary link gains and a cap on the transmission energy a node can spend on one packet.

Proof: The decision version of $\operatorname{MEAR}(V, s, t)$, DMEAR, can be described as follows. Given $(V, s, t)$, is there a feasible transmission schedule $S=$ $\left[\left(v_{1}, p_{1}\right), \cdots,\left(v_{w}, p_{w}\right)\right]$ for $(s, t)$, such that the total transmission energy $E(S) \triangleq \sum_{i=1}^{w} p_{i} \leq P$ ? Denote such an instance D-MEAR $(V, s, t, P)$.

First notice D-MEAR $\in$ NP, since given a transmission schedule, it can be verified in polynomial time if the schedule is feasible for $(s, t)$ and if the total energy consumption is at most $P$.

We show the NP-hardness part by reducing SC to DMEAR. The SC problem is defined as follows. Given set $S=\left\{v_{1}, \cdots, v_{n}\right\}$, and a collection of subsets of $S$, $\mathcal{C}=\left\{C_{1}, \cdots, C_{m}\right\}$. A set cover of $S$ is a subcollection $\mathcal{C}^{\prime} \subseteq \mathcal{C}$, such that every element of $S$ belongs to at least one member of $\mathcal{C}^{\prime}$. Is there a set cover $\mathcal{C}^{\prime}$ with $\left|\mathcal{C}^{\prime}\right| \leq B$ ? Denote such an instance $\operatorname{SC}(S, \mathcal{C}, B)$.

$$
\text { From } \mathrm{SC}(S, \mathcal{C}, B), \text { construct } \operatorname{D-MEAR}(V, s, t, P)
$$
where

$$
\begin{aligned}
V & =\left\{s, u_{1}, \cdots, u_{m}, C_{1}, \cdots, C_{m}, v_{1}, \cdots, v_{n}, t\right\} \\
P & =B(B+2)+n+1 .
\end{aligned}
$$

Call $\left\{v_{1}, \cdots, v_{n}\right\}$ the $S$ nodes, $\left\{C_{1}, \cdots, C_{m}\right\}$ the $\mathcal{C}$ nodes, $\left\{u_{1}, \cdots, u_{m}\right\}$ the $U$ nodes. Let $f(u)$ denote the subscript of node $u \in U \cup \mathcal{C}$, eg., $f\left(u_{i}\right)=i, f\left(C_{j}\right)=j$. 
Let $H=1$ and

$$
g_{j, i}= \begin{cases}1 & j=s, i \in U \\ 1 /(B+1) & j \in U, i \in \mathcal{C}, f(j)=f(i) \\ 1 & j \in \mathcal{C}, i \in S, i \in j \\ 1 / n & j \in S, i=t \\ 0 & \text { otherwise. }\end{cases}
$$

The construction of $\operatorname{D-MEAR}(V, s, t, P)$ is illustrated by Figure 3. We put an edge between nodes if and only if the gain between them is positive. Assume the transmission energy cap for each node is 1 . This is an important assumption for the proof. It forces all the $S$ nodes to transmit so that $t$ is able to successfully receive the packet. Now we only need to show

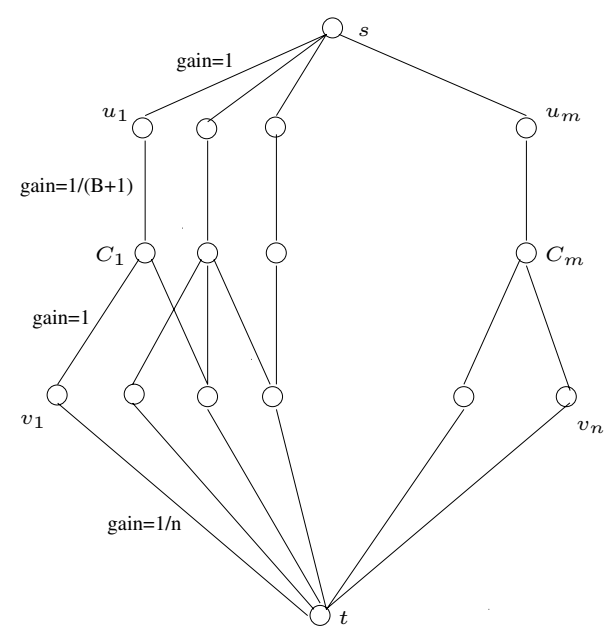

Fig. 3. D-MEAR instance reduced from SC.

$$
\operatorname{SC}(S, \mathcal{C}, B)=\text { Yes } \Longleftrightarrow \operatorname{D}-\operatorname{MEAR}(V, s, t, P)=\text { Yes. }
$$

If: Suppose we have a feasible transmission schedule for $(s, t)$ which consumes a total amount of energy of at most $P=B(B+2)+n+1$. Since $s$ must transmit with power 1; and each $S$ node must transmit with power 1, the total energy consumption used by $U$ nodes and $\mathcal{C}$ nodes is at most $B(B+2)$ in this schedule. Suppose $k$ nodes in $\mathcal{C}$ transmit. Since all $S$ nodes transmit and they can only receive the packet from these $k \mathcal{C}$ nodes, there must exist a set cover of $S$ with size at most $k$. Now we know that at least $k U$ nodes must transmit with power $B+1$ (to these $k \mathcal{C}$ nodes), consuming at least $k(B+1)$ energy. So we have

$$
k(B+1) \leq B(B+2)
$$

which means $k \leq B$ since $k$ is an integer. Therefore, the $k$ nodes in $\mathcal{C}$ that transmit correspond to a set cover of $S$, with size at most $B$.

Only if: Suppose $\mathcal{C}^{\prime}$ is a set cover with $\left|\mathcal{C}^{\prime}\right|=B$. The following transmission schedule is feasible for $(s, t)$.
First, source $s$ transmits with power 1, which enables all the $U$ nodes to receive the packet successfully. Suppose $U^{\prime} \subseteq U$ corresponds to $\mathcal{C}^{\prime}$ (i.e., the set of the subscripts of the nodes in $U^{\prime}$ is the same as that of the subscripts of the nodes in $\mathcal{C}^{\prime}$ ). Next, nodes in $U^{\prime}$ transmit with power $B+1$, which enables nodes in $\mathcal{C}^{\prime}$ to receive the packet successfully. Then, the nodes in $C^{\prime}$ transmit with power 1 . Since $\mathcal{C}^{\prime}$ covers all the elements of $S$, all $S$ nodes receive the packet successfully. Finally all $S$ nodes transmit with power 1 , each contributing $\frac{1}{n}$ unit of energy to $t$ 's reception, enabling $t$ to decode the packet successfully. Thus, the transmission schedule $S=$ $\left[(s, 1),[(u, B+1)]_{u \in U^{\prime}},[(C, 1)]_{C \in \mathcal{C}^{\prime}},\left[\left(v_{i}, 1\right)\right]_{i=1}^{n},(t, 0)\right]$ is a feasible transmission schedule for $(s, t)$; and the total energy cost is $B(B+2)+n+1$.

\section{Performance Analysis of Shortest Path Heuristic}

One natural heuristic for MEAR is to define the edge weight of $(j, i)$ as $H / g_{j, i}$ and apply any shortest path algorithm to find a path from $s$ to $t$ (without considering energy accumulation), then calculate the transmission powers with energy accumulation taken into account. Theorem 2 shows that this shortest path heuristic can perform very badly in the general graph model.

Theorem 2: In a general graph model, let $S P H$ denote the solution from the shortest path heuristic for an MEAR problem, and $O P T$ be the optimal solution. In the worst case, the energy cost $E(O P T) \in o(E(S P H))$. Proof: Consider $V=\{1, \cdots, n, n+1\}, s=1, t=$ $n+1, H=1$. The gain between any two nodes $(j, i)$ is $g_{j, i}=\frac{1}{|i-j|+\varepsilon}$, where $\varepsilon \in o(1)$ is an arbitrarily small positive number. Therefore the weight on edge $(j, i)$ equals $|i-j|+\varepsilon$. We show that $E(O P T) \in o(E(S P H))$ for problem $\operatorname{MEAR}(V, s, t)$ in this case.

First note that the shortest path found without accumulation is $s \rightarrow t$ directly. Thus, there is no leakage accumulation in $S P H$, resulting in the same energy expenditure as traditional $S P$. The total energy cost of $S P H$ is $n+\varepsilon$.

$$
E(S P H)=n+\varepsilon
$$

We first consider the following equation system on $e_{i}, i=1, \cdots, n$.

$$
\sum_{j=1}^{i} \frac{e_{j}}{i-j+1}=1, \quad i=1, \cdots, n
$$

It can be shown that the solution is:

$$
e_{i}=\int_{0}^{1}\left[\begin{array}{l}
t \\
i
\end{array}\right] d t, i=1, \cdots, n
$$

where $\left[\begin{array}{l}t \\ i\end{array}\right]=\frac{t(t+1)(t+2) \cdots(t+i-1)}{i !},\left[\begin{array}{l}t \\ 0\end{array}\right]=1$ (see [25] for details). It is easy to verify that $e_{i} \in[0,1], \forall i$ and $e_{i}$ is a non-increasing sequence. 
There are only $o(n)$ of the $e_{i}$ 's that are $\Theta(1)$. Suppose the opposite, i.e., there exist constants $c_{1}, c_{2}$ s.t. $c_{1} n$ of $e_{i}$ 's satisfy $e_{i}>c_{2}$. Then they must be $e_{1}, \cdots, e_{c_{1} n}$ since $e_{i}$ is non-increasing. Look at the $\left(c_{1} n\right)$ th equation in Equations 3,

$$
\begin{aligned}
1 & =\sum_{j=1}^{c_{1} n} \frac{e_{j}}{c_{1} n-j+1}>\sum_{j=1}^{c_{1} n} \frac{c_{2}}{c_{1} n-j+1} \\
& \in \Theta(\ln n)
\end{aligned}
$$

which is a contradiction. Therefore, among $e_{i}$ 's, $o(n)$ of them are $\Theta(1)$ and the other $O(n)$ of them are $o(1)$ (notice $\left.e_{i} \in[0,1], \forall i\right)$, so $\sum_{i=1}^{n} e_{i} \in o(n)$. Now consider schedule $S=\left[\left(i, p_{i}\right)\right]_{i=1}^{n+1}$ where $p_{i}=$ $e_{i}+\varepsilon, i=1, \cdots, n$ and $p_{n+1}=0$. Since

$$
\begin{aligned}
& \sum_{j=1}^{i} \frac{p_{j}}{i-j+1+\varepsilon}=\sum_{j=1}^{i} \frac{e_{j}+\varepsilon}{i-j+1+\varepsilon} \\
& \geq \sum_{j=1}^{i} \frac{e_{j}}{i-j+1}=1, \quad i=1, \cdots, n
\end{aligned}
$$

$S$ is a valid feasible schedule for $(s, t), E(O P T) \leq$ $E(S)$. Notice that

$$
E(S)=\sum_{i=1}^{n+1} p_{i}=n \varepsilon+\sum_{i=1}^{n} e_{i} \in o(n) .
$$

Theorem 2 follows.

\section{The Structure of Optimal Transmission Schedules}

In [3], the minimum-energy accumulative broadcast problem is divided into two subproblems. The subproblem of identifying the ordering in which the nodes transmit is found to be NP-complete and thus the main difficulty of the whole problem. Our MEAR problem can also be divided into two subproblems. The first is to determine which nodes should participate in the transmission schedule. The second is to specify the order in which the nodes transmit and their transmission powers. It turns out that once the first subproblem is solved, it is easy to determine the transmission order and the transmission power of each node sequentially. So the difficulty lies in the first subproblem.

Definition 2: A feasible schedule $S=\left[\left(v_{i}, p_{i}\right)\right]_{i=1}^{w}$ for problem $\operatorname{MEAR}(V, s, t)$ is a wavepath iff

1) no node transmits more than once, i.e., $\forall i \neq$ $j, v_{i} \neq v_{j}$, and

2) each node verifies the wavepath property, i.e.,

$$
\forall i, \quad \sum_{j=1}^{i-1} p_{j} g_{j, i}=H
$$

After each transmission, exactly one more node becomes capable of decoding the packet correctly; and each transmission uses the exact amount of power to make one more node able to decode the packet correctly.

Theorem 3: $\operatorname{A} \operatorname{MEAR}(V, s, t)$ problem always has an optimal schedule that is a wavepath.

Proof: Suppose the optimal schedule for $\operatorname{MEAR}(V, s, t)$ is $S=\left[\left(v_{i}, p_{i}\right)\right]_{i=1}^{w}$. It is easy to see $p_{w}=0$. If $v_{i}=v_{j}, i<j$, then obviously the schedule $S^{\prime}=\left[\left(v_{1}, p_{1}\right), \cdots,\left(v_{i}, p_{i}+\right.\right.$ $\left.\left.p_{j}\right), \cdots,\left(v_{j-1}, p_{j-1}\right),\left(v_{j+1}, p_{j+1}\right), \cdots,\left(v_{w}, p_{w}\right)\right]$

is also a feasible transmission schedule and $E\left(S^{\prime}\right)=E(S)$.

Now we prove the wavepath property part by showing that if $m>0$ nodes in $S$ do not verify the wavepath property, then it can be transformed into a schedule $S^{\prime}$ with $E\left(S^{\prime}\right)=E(S)$ and at most $m-1$ nodes in $S^{\prime}$ do not verify the wavepath property. Suppose $v_{i}$ is the last node in $S$ which does not verify the wavepath property, i.e.,

$$
\sum_{j=1}^{i-1} p_{j} g_{j, i}>H
$$

and $v_{i+1}, \cdots, v_{w}$ all verify the wavepath property. We can write the transmission power of $v_{i}, \cdots, v_{w-1}$ in schedule $S$ as functions of $p_{i-1}$ :

$$
\begin{aligned}
p_{i}= & \frac{1}{g_{i, i+1}}\left(H-\sum_{j=1}^{i-2} p_{j} g_{j, i+1}-p_{i-1} g_{i-1, i+1}\right) \\
\equiv & A_{i} p_{i-1}+B_{i} \\
p_{i+1}= & \frac{1}{g_{i+1, i+2}}\left(H-\sum_{j=1}^{i-2} p_{j} g_{j, i+2}-\right. \\
& \left.p_{i-1} g_{i-1, i+2}-p_{i} g_{i, i+2}\right) \\
= & \frac{1}{g_{i+1, i+2}}\left(H-\sum_{j=1}^{i-2} p_{j} g_{j, i+2}-\right. \\
& \left.p_{i-1} g_{i-1, i+2}-\left(A_{i} p_{i-1}+B_{i}\right) g_{i, i+2}\right) \\
\equiv & A_{i+1} p_{i-1}+B_{i+1} \\
= & \frac{1}{g_{w-1, w}}\left(H-\sum_{j=1}^{i-2} p_{j} g_{j, w}-p_{i-1} g_{i-1, w}\right. \\
p_{w-1} & \left.-p_{i} g_{i, w}-\cdots-p_{w-2} g_{w-2, w}\right) \\
= & \frac{1}{g_{w-1, w}}\left(H-\sum_{j=1}^{i-2} p_{j} g_{j, w}-p_{i-1} g_{i-1, w}\right. \\
& -\left(A_{i} p_{i-1}+B_{i}\right) g_{i, w}-\cdots \\
& \left.-\left(A_{w-2} p_{i-1}+B_{w-2}\right) g_{w-2, w}\right) \\
& \quad-1
\end{aligned}
$$




$$
\equiv A_{w-1} p_{i-1}+B_{w-1}
$$

Now define intervals $I_{i-1}, I_{i}, \cdots, I_{w-1}, I_{0}$ as follows.

$$
\begin{aligned}
I_{i-1} & =\left\{\pi \in \mathbb{R} \mid \sum_{j=1}^{i-2} p_{j} g_{j, i}+\pi g_{i-1, i}>H\right\} \\
I_{i} & =\left\{\pi \in \mathbb{R} \mid H-\sum_{j=1}^{i-2} p_{j} g_{j, i+1}\right. \\
\left.-\pi g_{i-1, i+1}>0\right\} & \\
I_{i+1}= & \left\{\pi \in \mathbb{R} \mid H-\sum_{j=1}^{i-2} p_{j} g_{j, i+2}-\pi g_{i-1, i+2}\right. \\
& \left.\cdots\left(A_{i} \pi+B_{i}\right) g_{i, i+2}>0\right\} \\
I_{w-1} & \left\{\begin{array}{r}
\pi \in \mathbb{R} \mid H-\sum_{j=1}^{i-2} p_{j} g_{j, w}-\pi g_{i-1, w} \\
-\left(A_{i} \pi+B_{i}\right) g_{i, w}-\cdots- \\
\left(A_{w-2} \pi+B_{w-2}\right) g_{w-2, w}>0
\end{array}\right\} \\
I_{0}=(0, \infty) &
\end{aligned}
$$

Note that $I_{0}$ is the set of values which if taken by $p_{i-1}$ let $v_{i-1}$ stay in $S ; I_{i-1}$ is the set of values which if taken by $p_{i-1}$ let $v_{i-1}$ not verify the wavepath property; each $I_{c}, i \leq c \leq w-1$ is the set of values which if taken by $p_{i-1}$ let $v_{c}$ continue to verify the wavepath property. These are all well-defined, open, infinite intervals because the constraints are all linear. Define interval $I$ as the intersection of them: $I=\left(\bigcap_{j=i-1}^{w-1} I_{j}\right) \cap I_{0}$. Since at least $p_{i-1}$ is in $I, I$ is also an open infinite interval.

Now write $E(S)$ as a linear function of $p_{i-1}$ :

$$
\begin{aligned}
E(S) & =\sum_{j=1}^{i-2} p_{j}+p_{i-1}+\sum_{j=i}^{w-1}\left(A_{j} p_{i-1}+B_{j}\right)+0 \\
& \equiv A p_{i-1}+B
\end{aligned}
$$

We show that $A=0$. Suppose instead $A>0$. There exists $\pi \in I$ such that $\pi<p_{i-1}$ and the schedule $S^{\prime}=\left[\left(v_{1}, p_{1}\right), \cdots,\left(v_{i-2}, p_{i-2}\right),\left(v_{i-1}, \pi\right),\left(v_{i}, A_{i} \pi+\right.\right.$ $\left.\left.B_{i}\right), \cdots,\left(v_{w-1}, A_{w-1} \pi+B_{w-1}\right),\left(v_{w}, 0\right)\right]$ is a feasible transmission schedule and $E\left(S^{\prime}\right)<E(S)$. This contradicts that $S$ is an optimal schedule. The argument is similar for the case $A<0$.

Now we show how to transform $S$, which has $m>0$ nodes in the schedule violating the wavepath property, to $S^{\prime}$, which has the same energy consumption as $S$ and only $m-1$ nodes violate the wavepath property. Denote the left boundary of interval $I$ by $\ell(I)$. Note that $\ell(I)<p_{i-1}<\infty$. There are three cases.
1) $\ell(I)=\ell\left(I_{j_{1}}\right)=\cdots=\ell\left(I_{j_{x}}\right), i \leq j_{1}<\cdots<$ $j_{x} \leq w-1$. Transform $S$ into $S^{\prime}$ by setting $p_{i-1}=\ell(I)$ and changing $p_{j}, i \leq j \leq w-1$ according to the formulae $p_{j}=A_{j} p_{i-1}+B_{j}$, and removing those $\left(v_{j}, p_{j}\right)$ pairs which have $p_{j}=0$ (i.e., $\left.\left(v_{j_{1}}, p_{j_{1}}\right), \cdots,\left(v_{j_{x}}, p_{j_{x}}\right)\right)$. It is easy to verify that $S^{\prime}$ is a feasible transmission schedule and $E\left(S^{\prime}\right)=E(S)$. Now note that if there are still $m$ nodes that do not verify the wavepath property, then the last such node must continue to be $v_{i}$. Use the arguments above again (i.e., write $p_{j}, j \geq i$ and $E\left(S^{\prime}\right)$ as linear functions of $p_{i-1}$, define the intervals, and $A=0)$. If $\ell(I)$ still falls in this case, repeat the arguments again. Finally $\ell(I)$ must fall in the other cases since in each repetition, some $\left(v_{j}, p_{j}\right)$ pairs are removed; and some intervals do not have a left boundary (eg. $I_{i}$ ).

2) $\ell(I)=\ell\left(I_{i-1}\right)$. Transform $S$ into $S^{\prime}$ by setting $p_{i-1}=\ell(I)=\ell\left(I_{i-1}\right)$ and changing $p_{j}, i \leq j \leq$ $w-1$ according to the formulae $p_{j}=A_{j} p_{i-1}+B_{j}$. It is easy to verify that $S^{\prime}$ is a feasible transmission schedule and $E\left(S^{\prime}\right)=E(S)$. Note in $S^{\prime} v_{i}$ verifies the wavepath. So at most $m-1$ nodes do not verify the wavepath property.

3) $\ell(I)=\ell\left(I_{0}\right)=0$. Transform $S$ into $S^{\prime}$ by setting $p_{i-1}=0$, removing $\left(v_{i-1}, p_{i-1}\right)$, and changing $p_{j}, i \leq j \leq w-1$ according to the formulae $p_{j}=$ $A_{j} p_{i-1}+B_{j}$. It is easy to verify that $S^{\prime}$ is a feasible transmission schedule and $E\left(S^{\prime}\right)=E(S)$. At most $m-1$ nodes in $S^{\prime}$ do not verify the wavepath property.

Thus any optimal schedule of $\operatorname{MEAR}(V, s, t)$ can be iteratively transformed to a schedule where every node verifies the wavepath property.

Theorem 4: Given the $\operatorname{MEAR}(V, s, t)$ problem, and the set of participating nodes $U \subset V, s, t \notin U$ in an optimal transmission schedule $S=\left[\left(v_{i}, p_{i}\right)\right]_{i=1}^{w}$ which is a wavepath, i.e., $\left\{v_{i}\right\}_{i=2}^{w-1}=U$ ( $S$ itself is not given), there is a polynomial time algorithm to find $S$.

Proof: We provide an algorithm ORDER as in Algorithm 1 which output the integral optimal transmission schedule $S$ when $U$ is given. It is easy to verify that ORDER runs in $O\left(w^{2}\right)$ time and based on Theorem 3, it is correct.

Note that in Theorem 4, node participation is predetermined, i.e., all nodes in $U$ must be in $S$ and only the nodes in $U$ plus $s, t$ can be in $S$.

\section{E. A Heuristic RPAR for $\operatorname{MEAR}(V, s, t)$}

In this section, we present our Relay PAth Routing heuristic, RPAR, for identifying the energy efficient accumulative relay route of the $\operatorname{MEAR}(V, s, t)$ problem. Through simulations it is shown that RPAR can achieve 


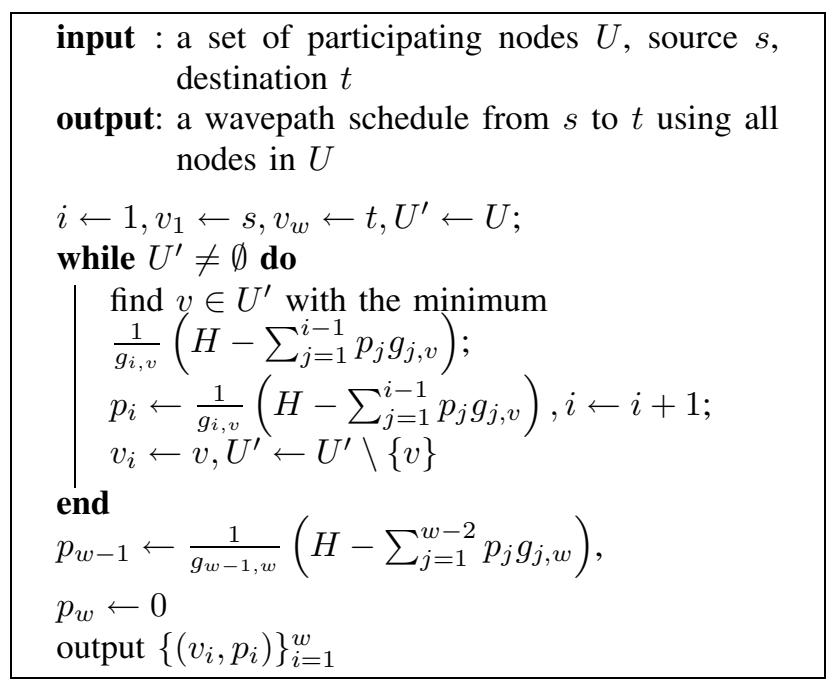

Algorithm 1: $\operatorname{OrDER}(U, s, t)$

more than $30 \%$ improvement over SP, the traditional shortest path algorithm. Also the performance of RPAR is very close to the optimal, both in the average case and in the worst case as shown in Section IV-A.

RPAR grows a tree $T$ rooted at $s$ by connecting a node to $T$ in each iteration, starting with $\{s\}$, until $t$ is added to $T$. Define a dynamic cost function $c(u, v)$ as the energy consumption of a successful transmission from $u$ to $v$. Define function $e(v)$ on node $v$ as the total energy usage of a transmission schedule from $s$ to $v$ if the unique path from $s$ to $v$ in $T$ is followed. In the description of Algorithm 2, $\pi(v)$ denotes the parent of node $v$ in $T, V(T)$ denotes the vertex set of $T, E(T)$ denotes the edge set of $T$, and $p(v)$ denotes the unique path from $s$ to $v$ in $T$, i.e. $p(v)=\left[\left(v_{1}, v_{2}\right),\left(v_{2}, v_{3}\right), \cdots,\left(v_{w-1}, v_{w}\right)\right]$ where $v_{1}=s, v_{w}=v$ and $\forall i<w,\left(v_{i}, v_{i+1}\right) \in E(T)$.

The output is an energy efficient transmission schedule from $s$ to $t$. It is clear from the description of Algorithm 2 that a node $u$ can transmit to its next hop $v$ with energy smaller than $H / g_{u, v}$ (except for source $s$ ), which forms the basis of better energy efficiency in a $A R$ network than a TM network.

The execution of $\operatorname{RPAR}(V, s, t)$ assigns a unique parent for each node that is added to $T$. It follows that $T$ is a tree. So for given source $s$ and destination $t$, RPAR yields a loop free path. It can be shown that RPAR has time complexity $O\left(V^{3}\right)$. (For each iteration to add one node to $T$, it needs $O\left(V^{2}\right)$ computation time.)

\section{F. Analysis of Energy Efficiency of $k$-Relay}

In this section, we derive a lower bound on the energy efficiency of $k$-Relay routing. By the same derivation, we show the existence of an algorithm that achieves the bounded approximation ratio. Note that this bound is only interesting when $k$ is small.
input : node set $V$, source $s \in V$, destination $t \in$ V
output: a transmission schedule from $s$ to $t$

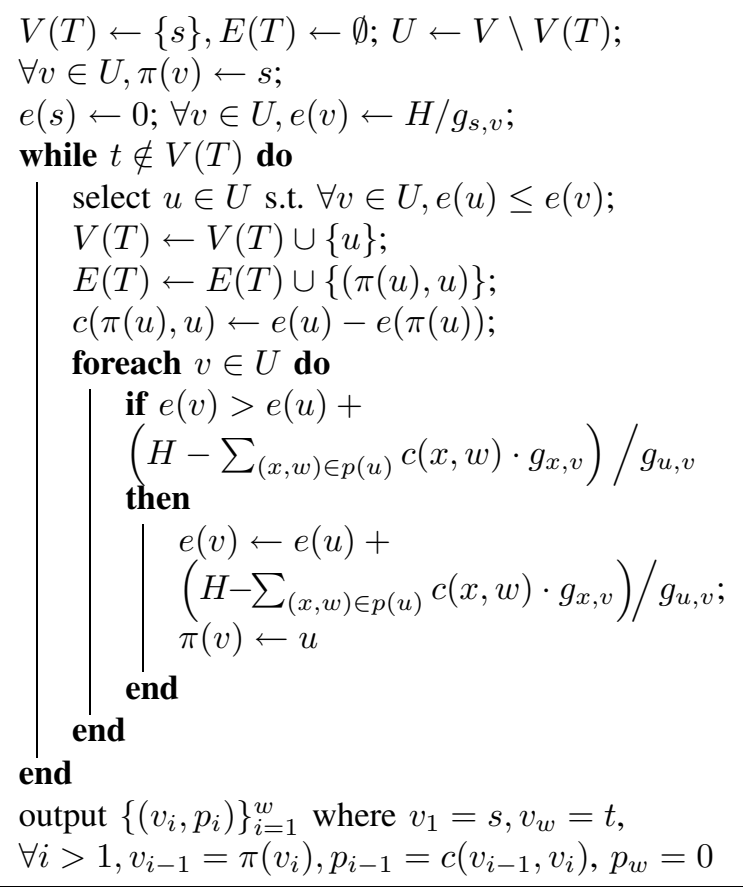

Algorithm 2: $\operatorname{RPAR}(V, s, t)$

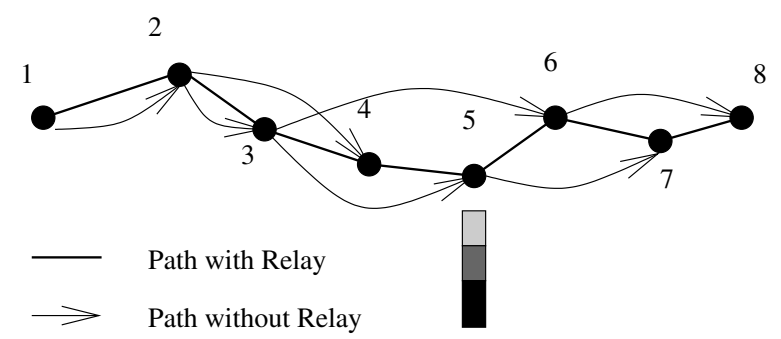

Fig. 4. For source destination pair 1 and 8 , let the optimal 3 -Relay path be $[1,2, \cdots, 8]$. The directed edges represent the edges $(A(i), i)$ if leakage of node $A(i)$ at node $i$ is at least $\frac{1}{3} H$. For example, $A(2)=1, A(6)=3$. The sequence $1,2,3,6,8$ forms a directed path from 1 to 8 in the $T M$ model.

Theorem 5: Given a set of nodes $V$, source $s \in V$ and destination $t \in V$, the energy of the optimal $k$ Relay path is at least $\frac{1}{k}$ of the output given by the SP algorithm.

\section{Proof:}

Let $S=\left[\left(v_{1}=s, p_{1}\right), \cdots,\left(v_{l}=t, p_{\ell}\right)\right]$ be the optimal $k$-Relay transmission schedule for $(s, t)$, and let $E(S)=\sum_{i=1}^{i=\ell-1} p_{i}$ be the total energy consumption of $S\left(p_{\ell}=0\right)$. By the definition of $k$-Relay, node $i$ can accumulate energy from transmissions of nodes $i-k, i-$ $k+1, \cdots, i-1$, which sum to $H$. Thus, for any node 
$i>1$, at least one node among $i-k, i-k+1, \cdots, i-1$ has a leakage of at least $\frac{1}{k} H$ on $i$. Let $A(i)$ denote this node. We now show that $S^{*}=\left[\left(v_{i}, p_{i}^{*}\right)\right]_{i=1}^{w}$, where $v_{1}=s, v_{w}=t, p_{i}^{*}=k p_{i}$ and $\forall i>1, v_{i-1}=A(i)$, is a feasible transmission schedule for 1-Relay routing.

First, $S^{*}$ is well-defined, since for any node $i>1$, $A(i)$ exists and $A(i)<i$. The schedule $S^{*}$ can be found by determining $t, A(t), A(A(t)), A(A(A(t))), \cdots$. Now by definition of $A(i)$, the transmission power $p_{i-1}^{*}$ of every node $i-1$ is enough for the next node $i$ to correctly decode the packet. Therefore, $S^{*}$ is feasible for 1-Relay routing.

Since $E(S)$ is at least $\frac{1}{k}$ fraction of the total energy consumption of $S^{*}$, which is at least the shortest path energy (minimum among all 1-Relay paths), Theorem 5 follows.

Theorem 5 places a lower bound on the energy efficiency of $k$-Relay routing, i.e., the shortest path heuristic provides an approximation of factor $k$. When $k=n$ (accumulation allowed from any previous transmitting node), the upper bound of the performance of this heuristic is $n$. In Section III-C, we have shown that the shortest path heuristic can perform arbitrarily badly (with a super-constant approximation), which provides a lower bound of the heuristic performance. However, when $k$ is small, eg. $k=2$, the heuristic can provide 2-approximation guarantee.

\section{Simulation Results}

In this section, we evaluate the performance of RPAR by first comparing it to the optimal solution OPTRelay in small size networks and then to SP in larger networks through simulations. We consider the aggregate transmission energy consumption of the paths (defined in Section II) as the performance metric. Nodes are randomly distributed in a stationary network with size $1000 m \times 1000 m$. In all the simulations, the power attenuation exponent $\alpha$ is set to 2 .

\section{A. Comparison Between RPAR, SP and OPTRelay}

We first demonstrate the performance of the heuristic RPAR in approximating the optimal solutions, and compare it with that of SP. For networks with $n=$ $2,3, \cdots, 26$ nodes, we measure the average case approximation ratios and the worst case approximation ratios of RPAR and SP. The approximation ratio is defined as the total transmission energy of the schedule output by RPAR divided by that of an optimal schedule, and the same for SP. The optimal schedule is found by brute force search. For each $n$-node network, we study the approximation ratio for all the source-destination pairs in the network, and plot the average and the worst case approximation ratios in Figure 5. It shows that RPAR heuristic is very close to the OPTRelay. Its approximation ratio is no more than than 1.1 in the worse cases, and even less in average case which is around 1.01. On the contrary, the SP algorithm, deviates from the optimal significantly, in the worst case as well as in the average case.

Recall that Theorem 5 establishes that both RPAR and SP are $k$ factor approximation algorithms for the minimum energy accumulative $k$-Relay routing. The simulation shows that the actual performance of RPAR is much better than that of SP. It is interesting to see that the approximation ratio of either RPAR or SP does not exceed 2 , even for unlimited $k$, indicating that the performance upper bounds can be significantly improved. In the following discussions, we compare the performance of RPAR with that of SP for larger networks in various settings. We repeat each simulation 50 times and compute the average as our simulation result.

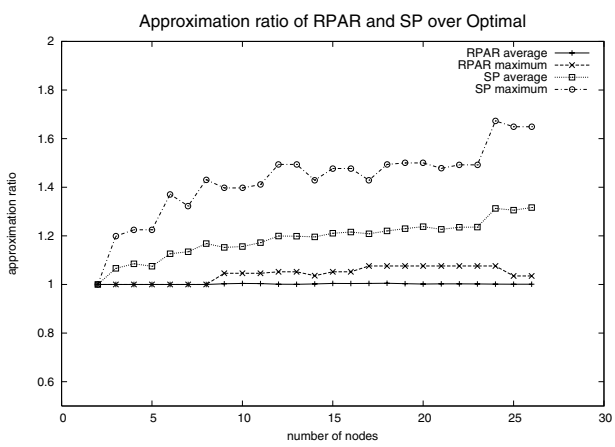

Fig. 5. Average and worst case performance of RPAR and SP on small networks.

\section{B. Impact of Nodes Density}

In this set of simulations, we investigate the effect of node density on the performance of RPAR. A set of $50,100,150,200,250,300,350$ or 400 nodes are randomly distributed in a $1000 \mathrm{~m} \times 1000 \mathrm{~m}$ plane. For each run of the simulation, 20 source-destination pairs are randomly selected, and we measure the total energy consumption of the schedules output by RPAR over that of SP. The results are illustrated in Figure 6. When the network density increases the ratio decreases which implies the energy saving of RPAR over SP increases. For a randomly selected pair, the expectation of the Euclidean distance between them remains the same when the density increases. However the path generated by RPAR (as well as by SP) will have more hops when the density increases. Thus, on one hand, the energy consumption of both schedules from RPAR and SP will decrease when the the density increases. On the other hand, the energy consumption of the RPAR schedule decreases even faster since more nodes generate leakages 
to other nodes in the network and moreover, the distance between each hop decreases which enable the nodes to benefit more from the leakages. This explains the observed trend in Figure 6.

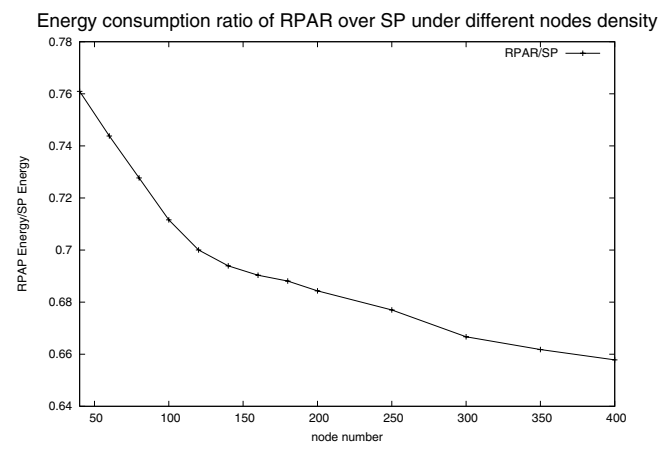

Fig. 6. Energy saving of RPAR over SP increases when the network density increases.

\section{Impact of the Number of Relay Levels}

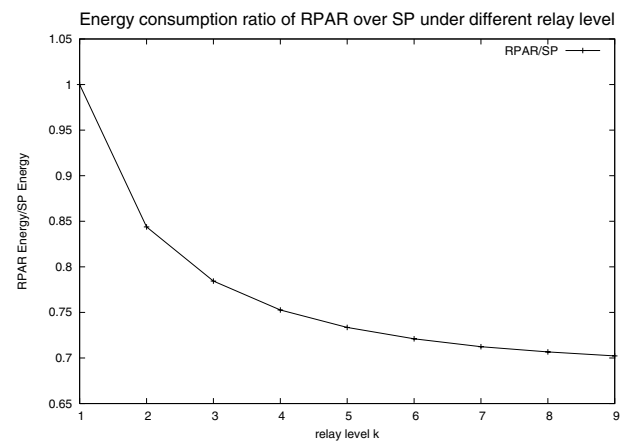

Fig. 7. Energy saving of RPAR over SP under different relay levels. Larger relay level leads to more energy saving, but the improvement becomes less when $k$ increases.

In this simulation, we study the performance of $k$ Relay $A R$ for different relay levels. We consider a random network of 200 nodes. For each relay level $k=1,2, \cdots, 9$, we plot the average energy consumption ratio of the schedules output by RPAR to that of SP for 20 random source destination pairs. The results are presented in Figure 7. Note that 1-Relay scheme is actually the traditional routing without accumulative relay. Thus, the ratio of 1-Relay RPAR over SP is simply 1 . When the relay level $k$ increases, the energy ratio of RPAR over SP decreases, but rate of decreasing slows down. Figure 7 shows that larger relay levels provides more energy saving, however, most energy saving of RPAR is achieved under small relay levels. This is an encouraging result, since the limited computational power and buffer size of wireless nodes as well as the complexity of coding schemes puts a limitation on the relay level in practical systems.

\section{IMPLEMENTATION ISSUES}

For the proposed scheme to be successfully implemented two main issues need to be addressed. First, the nodes should be able to compute the wavepath. Second, they should be able to implement the accumulative relaying. The first part can be implemented in a centralized manner, where one or multiple nodes gather the information about the network topoplogy and then run the RPAR algorithm. The distributed implementation of the RPAR algorithm requires further investigation specially when we consider mobility [26]. In this section, we assume that the wavepath was already established and we focus on the implementation of the accumulative relaying.
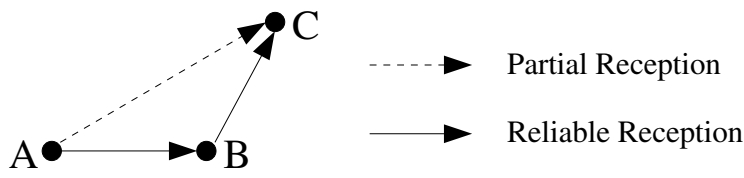

Fig. 8. Packet relaying.

To be able to correctly implement the accumulative relaying, each node should be capable of the following tasks.

1) Reliably identify each received packet by using a strong modulation/coding of the packet header even if the payload cannot be decoded. The goal here is to distinguish between the packets and to group them if they are copies of the same original packet. This issue can be dealt with by including in the header enough information for unique identification of the packet, and then encoding the packet header using a forward error correction code. The packet header should contain the following information:

- MAC_SRC_ADDR: source address at the link layer (address of the relay node sending this packet);

- MAC_DST_ADDR: destination address at the link layer (address of the relay node who is the immediate destination of this transmission);

- NET_SRC_ADDR: network address of the node that generated the packet;

- SN: a sequence number generated by the network source node, to uniquely identify a packet and all its relayed copies.

In Figure 8 , node $C$ can match the two copies from $A$ and $B$ by looking at the NET_SRC_ADDR and 
SN fields. Node $C$ should also be able to decode the header of the packet sent by $A$ even if it is not capable of decoding its payload. One approach to realize it is to use a forward error correction code. Using a good error correction code can provide the coding gain necessary to reach nodes within twice the range of the data part of the packet. If the power attenuation factor is taken to be equal to 2 , then it is enough to use a code with a gain of $20 \log _{10}(2)=6 d B$. The simplest code that can be used is a repetition code, however LDPC and turbo codes provide better gain for the same redundancy level [27], [28] but require more computation for decoding. The tradeoff between transmission energy and energy cost of decoding has to be considered to determine the best coding strategy.

2) Be able to store the partially received packet. At the MAC layer, the node should store all received packets corresponding to the same NET_SRC_ADDR and SN, until receiving a copy of such a packet with the MAC_DST_ADDR corresponding to the MAC address. Then the node can attempt to decode such packet and send an acknowledgment if successful. All the old copies of a packet will be discarded from the MAC memory when the packet is successfully decoded.

3) Be able to combine the various copies and correctly decode the packet. The data part of each packet is encoded with an error correction code that achieves a very low bit error rate for the considered power threshold. This implicitly implies that the rate of such code is below the Shannon capacity limit for the power threshold and noise level. When combining multiple copies of each packet, one can ask if such copies need to be encoded specially. In the case of the wideband regime it was shown in [3] that a simple repetition code provides optimal performance in terms of energy saving. This means that there is no advantage in using a complex re-coding scheme when forwarding a packet. This result is basically due to the fact that the capacity of the channel is proportional to the signal power for large bandwidth. Therefore the receiver can combine the stored copies of each packet by combining the different copies of each bit by, for example, computing an average of the real valued estimates.

4) Be able to prevent interference at all targeted neighboring nodes. Our target scenario is a low-load network where energy is the critical constraint. This is typically the case for sensor networks with duty cycles below $1 \%$. If the net- work load is not low, and if an IEEE802.11like MAC protocol is used, then the RTS/CTS collision avoidance mechanism should be modified to prevent interference at overhearing nodes. This can be done by using a forward error correction code for the RTS/CTS packets to cover all the area where overhearing nodes might be located.

5) Online power control and retransmissions. At each transmission, the sending node estimates the required power level for the receiver using the RTS/CTS handshake. The CTS packet includes the required power level and takes into account the previously accumulated energy. If the packet cannot be successfully decoded, the retransmission is done at a power level freshly estimated through the RTS/CTS exchange.

\section{CONCLUSIONS}

In this paper, we investigated a novel approach to energy saving for unicast communication under the model where nodes can partially overhear packets. This is feasible even with today's RF chips that allow multirate/coding/modulation communication. In search for simple and optimal relaying strategies, we introduced the notion of wavepath and showed that any minimum energy schedule can be transformed into a wavepath. We developed a heuristic to build an energy efficient wavepath and showed through simulation that significant energy saving can be achieved. We have also shown that under a general propagation model the classical shortest path approach can be arbitrarily bad in comparison with an optimal approach (and our heuristic).

\section{REFERENCES}

[1] J. G. Proakis, Digital Communications, 4th ed. McGraw Hill, 2001.

[2] L. G. Manish Agarwal, Joon Ho Cho and J. Wu, "Energy efficient broadcast in wireless ad hoc networks with hitch-hiking," in INFOCOM 2004, March 2004

[3] I. Maric and R. Yates, "Efficient multihop broadcast for wideband systems," in Allerton Conference on Communications, Control and Computing, October 2002.

[4] L. M. Feeney and M. Nilson, "Investigating the energy consumption of a wireless network interface in an ad hoc networking environment," in INFOCOM 2001, March 2001.

[5] K. Sohrabi, J. Gao, V. Ailawadhi, and G. Pottie, "Protocols for self-orginization of a wireless sensor network," in IEEE Personal Communications Magazine, October 2000, pp. 16-27.

[6] S. Singh, M. Woo, and C. Raghavendra, "Poweraware routing in mobile ad hoc networks," in MOBICOM 1998, 1998, pp. 181-190. [Online]. Available: citeseer.nj.nec.com/singh98poweraware.html

[7] J.-H. Chang and L. Tassiulas, "Energy conserving routing in wireless ad-hoc networks," in INFOCOM 2000, March 2000.

[8] V. Rodoplu and T. H. Meng, "Minimum energy mobile wireless networks," IEEE Journal on Selected Areas in Communications, vol. 17, no. 8, pp. 1333-1344, August 1999.

[9] S. Banerjee and A. Misra, "Minimum energy paths for reliable communication in multi-hop wireless networks," in MOBIHOC 2002, June 2002. 
[10] M. Youssef, M. Younis, and K. Arisha, "A constrained shortestpath energy-aware routing algorithm for wireless sensor networks," in IEEE WCNC 2002, March 2002.

[11] V. Srinivasan, C. E. Chiasserini, P. Nuggehalli, and R. Rao, "Optimal rate allocation and traffic splits for energy efficient routing in ad hoc networks," in INFOCOM 2002, June 2002.

[12] P. Manzoni and J.-C. Cao, "A performance comparison of energy consumption for mobile ad hoc routing protocols," in Proc. of the 8th International Symposium on Modeling, Analysis and Simulation of Computer and Telecommunication Systems, 2000.

[13] A. Michail and A. Ephremides, "Energy efficient routing for connection-oriented traffic in ad hoc wireless networks," in PIMRC'02: Proc. of the 11th IEEE International Symposium on Personal, Indoor and Mobile Radio Communications, 2002.

[14] S. Doshi, S. Bhandare, and T. X. Brown, "An on-demand minimum energy routing protocol for a wireless ad hoc network," in ACM Mobile Computing and Communications Review, July 2002.

[15] J. Chen, R. Rajaraman, and R. Sundaram, "Meet and merge: Approximation algorithms for confluent flows," in STOCS 2003, 2003.

[16] T. M. Cover and J. A. Thomas, Elements of information theory. Wiley-Interscience, 1991.

[17] E. C. V. der Meulen, "A survey of multi-way channels in information theory," in IEEE Transactions on Information Theory, vol. 23, January 1977 , pp. 1-37.

[18] T. M. Cover and A. E. Gamal, "Capacity theorems for the relay channel," in IEEE Transactions on Information Theory, vol. 25, no. 5, September 1979, pp. 572-584.

[19] M. Gastpar and M. Vetterli, "On the capacity of wireless networks: The relay case," in INFOCOM 2002, June 2002.

[20] P. Gupta and P. R. Kumar, "Towards an information theory of large networks: An achievable rate region," in IEEE Transactions on Information Theory, vol. 49, no. 8, August 2003, pp. 18771894.

[21] A. Reznik, S. R. Kulkarni, and S. Verdu, "Capacity and optimal resource allocation in the degarded gaussian channel with multiple relays," in 40th Allerton Conference on Communication, Control and Computing, October 2002.

[22] F. M. J. Willems, "Information theorectical results for the discrete memeoryless multiple access channel," Ph.D. dissertation, de Wetenschappen Proefschrift, Katholieke Univeristeit Leuven, Leuven, Belgium, 1982.

[23] T. Rappaport, Wireless Communications: Principles and Practices. Prentice Hall, 1996.

[24] U. Feige, "A threshold of $\operatorname{lnn}$ for approximating set cover," in Journal of the ACM, 1998.

[25] J. Chen, L. Jia, X. Liu, G. Noubir, , and R. Sundaram, "Minimum energy accumulative routing in wireless networks," College of Computer \& Information Science, Northeastern University, Tech. Rep., July 2004.

[26] G. Lin, G. Noubir, and R. Rajaraman, "Mobility models for ad hoc networks," in INFOCOM 2004, March 2004.

[27] T. Richardson, M. Shokrollahi, and R. Urbanke, "Design of capacity-approaching irregular low-density parity-check codes," in IEEE Transactions on Information Theory, vol. 47, no. 2, February 2001

[28] C. Berrou, A. Glavieux, and P. Thitimajshima, "Near shannon limit error-correcting coding and decoding: Turbo codes," in ICC, 1993. 